\title{
Rapid measurement of SARS-CoV-2 spike T cells in whole blood from vaccinated and naturally infected individuals
}

\author{
Anthony T. Tan, ${ }^{1}$ Joey M.E. Lim, ${ }^{1}$ Nina Le Bert, ${ }^{1}$ Kamini Kunasegaran, ${ }^{1}$ Adeline Chia, ${ }^{1}$ Martin D.C. Qui, ${ }^{1}$ Nicole Tan, ${ }^{1}$ Wan Ni Chia, ${ }^{1}$ \\ Ruklanthi de Alwis, ${ }^{1,2}$ Ding Ying, ${ }^{3,4,5}$ Jean X.Y. Sim, ${ }^{6}$ Eng Eong Ooi, ${ }^{1}$ Lin-Fa Wang, ${ }^{1}$ Mark I-Cheng Chen, ${ }^{3}$ Barnaby E. Young, ${ }^{3,4,5}$ \\ Li Yang Hsu, Jenny G.H. Low, ${ }^{1,6}$ David C. Lye, ${ }^{3,4,5,8}$ and Antonio Bertoletti ${ }^{1,9}$ \\ 1Programme in Emerging Infectious Diseases, Duke-NUS Medical School, Singapore. ${ }^{2}$ Viral Research and Experimental Medicine Centre (ViREMiCS), SingHealth Duke-NUS Academic Medical Centre, \\ Singapore. ${ }^{3}$ National Centre for Infectious Diseases (NCID), Singapore. ${ }^{4}$ Department of Infectious Diseases, Tan Tock Seng Hospital, Singapore. ${ }^{5}$ Lee Kong Chian School of Medicine, Singapore. ${ }^{6}$ Department \\ of Infectious Diseases, Singapore General Hospital, Singapore. ${ }^{7}$ Saw Swee Hock School of Public Health, National University of Singapore and National University Health System, Singapore. ${ }^{8}{ }^{Y}$ ong Loo Lin \\ School of Medicine, Singapore. ${ }^{9}$ Singapore Immunology Network, A*STAR, Singapore.
}

\begin{abstract}
Defining the correlates of protection necessary to manage the COVID-19 pandemic requires the analysis of both antibody and T cell parameters, but the complexity of traditional tests limits virus-specific $T$ cell measurements. We tested the sensitivity and performance of a simple and rapid SARS-CoV-2 spike protein-specific T cell test based on the stimulation of whole blood with peptides covering the SARS-CoV-2 spike protein, followed by cytokine (IFN- $\gamma$, IL-2) measurement in different cohorts including BNT162b2-vaccinated individuals $(n=112)$, convalescent asymptomatic and symptomatic COVID-19 patients ( $n=$ 130), and SARS-CoV-1-convalescent individuals $(n=12)$. The sensitivity of this rapid test is comparable to that of traditional methods of T cell analysis (ELISPOT, activation-induced marker). Using this test, we observed a similar mean magnitude of T cell responses between the vaccinees and SARS-CoV-2 convalescents 3 months after vaccination or virus priming. However, a wide heterogeneity of the magnitude of spike-specific T cell responses characterized the individual responses, irrespective of the time of analysis. The magnitude of these spike-specific $\mathrm{T}$ cell responses cannot be predicted from the neutralizing antibody levels. Hence, both humoral and cellular spike-specific immunity should be tested after vaccination to define the correlates of protection necessary to evaluate current vaccine strategies.
\end{abstract}

\section{Introduction}

SARS-CoV-2, the etiological agent of COVID-19, has spread worldwide, resulting in a global health and economic crisis that mass vaccinations are trying to resolve. The host's capacity to be protected from viral infection or from the development of severe diseases requires a coordinated activation of different components of the immune system that ultimately leads to the production of neutralizing and antigen-binding antibodies and antiviral $\mathrm{T}$ cells. Evidence that antibodies and $\mathrm{T}$ cells are required for protection has been found in monkeys challenged with SARS-CoV-2 (1). Similarly, antibodies and $\mathrm{T}$ cells are present in the majority of SARS-CoV-2-infected individuals who have controlled infection without severe symptoms $(2-6)$, and a robust $\mathrm{CD}^{+} \mathrm{T}$ cells

Conflict of interest: ATT, NLB, and AB are co-inventors on a patent titled "Method to Monitor SARS-Cov-2-Specific T Cells in Biological Samples" (patent application no. 10202010411P). WNC and LFW, in a sublicensing agreement with GenScript, are co-inventors on a patent titled "SARS-CoV-2 surrogate neutralization test based on antibody-mediated blockage of ACE2 spike protein binding" (patent application no. $17 / 220,043)$. AB has received personal fees from Oxford Immunotech and QIAGEN Copyright: @ 2021, American Society for Clinical Investigation.

Submitted: June 15, 2021; Accepted: July 20, 2021; Published: September 1, 2021

Reference information: / Clin Invest. 2021;131(17):e152379.

https://doi.org/10.1172/JCl152379. response is associated with mild disease in oncological patients with humoral defects (7).

Recently developed SARS-CoV-2 vaccines that protect more than $90 \%$ of the vaccinated individuals from severe COVID-19 can induce spike-specific antibodies and T cells $(8,9,10)$. However, it is not entirely clear what level of antibodies and/or T cells is necessary to confer such protection or whether differences in antibody and $\mathrm{T}$ cell levels in fact exist in vaccinated persons. Efforts to define the protective threshold of antibodies through mathematical modeling (11) have shed some light on this issue, but such work on $\mathrm{T}$ cell responses has so far been absent. Although experimental data have shown that high levels of neutralizing antibodies can be sufficient to protect against experimental infection, lower levels require the presence of $\mathrm{T}$ cells (1). Neutralizing antibody titers are, however, extremely heterogeneous after natural infection (12), and while most of the new SARS-CoV-2 vaccines induce high neutralizing antibody levels $(10,13)$, their persistence over time needs to be evaluated. Instead, virus-specific T cells appear to persist for a long time after viral clearance (i.e., 17 years after SARS-CoV-1 infection), and detection of SARS-CoV-2-specific T cells in patients with COVID-19 with waning antibody titers has been reported by different groups $(5,4,14,15)$. Furthermore, the protective role of spike-specific $\mathrm{T}$ cells in vaccinated individuals 
has also been highlighted by a recent analysis of the early profile of spike-specific immunity (16).

We think that the correlates of protection induced by vaccinations should therefore be derived from large prospective studies in which the levels of both antibodies and $\mathrm{T}$ cells are measured. However, although tests for antibodies are routinely performed, the technical complexity of SARS-CoV-2 T cell measurements has so far limited this analysis, with some exceptions (17), to a small number of individuals characterized in a few specialized laboratories. This is because $\mathrm{T}$ cells specific for a defined pathogen constitute a minuscule fraction of total $\mathrm{T}$ cells (often less than 1\%-3\%) present in the blood and can be distinguished mainly by complex functional assays that preserve the viability of the $\mathrm{T}$ cells during the assay. In addition, methods that are technically simple and do not require complex laboratory equipment, like the ELISPOT assay, need to be performed in cells that have been purified from whole blood. This introduces into the assay the lengthy and technically demanding processes of PBMC separation. Other assays that can directly measure the frequency and function of virus-specific $\mathrm{T}$ cells through expression of activation markers or cytokine production necessitate more complex equipment (i.e., a flow cytometer) and highly specialized personnel that might not be available in every routine diagnostic laboratory.

A possible rapid and simple alternative to these methods is the direct addition of stimulatory antigens or peptides to whole blood that induce the secretion of cytokines (usually IFN- $\gamma$ ) in plasma, which is subsequently quantified. This assay is routinely applied in the diagnosis of active tuberculosis (18), and it has also been shown to measure the presence of SARS-CoV-2-specific T cells in asymptomatic (5) and symptomatic SARS-CoV-2-infected $(19,20)$ patients. However, to our knowledge, its accuracy and validation have not been adequately analyzed over time in individuals who have been vaccinated against SARS-CoV-2, and only responses immediately after vaccination have been tested (16). Therefore, in this study, we applied a range of cellular methods to measure SARS-CoV-2 $\mathrm{T}$ cell responses in individuals vaccinated with the pre-fusion-stabilized, full-length SARS-CoV-2 spike protein (BNT162b2) or in those naturally infected with SARS-CoV-2. We demonstrated that the detection and relative quantification of spike-specific $\mathrm{T}$ cells in vaccinated individuals can be easily and rapidly achieved through the simple addition of spike peptide pools to whole blood. Utilization of different peptide pools to stimulate whole blood provides the flexibility to derive rapid information about the kinetics and magnitude of spike-specific $\mathrm{T}$ cell responses induced by vaccination and compare them with those present in convalescent individuals.

\section{Results}

Rapid quantification of SARS-CoV-2 spike-specific T cells by direct peptide stimulation of whole peripheral blood. We characterized the initial kinetics of spike-specific T cells induced by 2 doses of the mRNA vaccine BNT162b2 over a 51-day period using different methods of antigen-specific $\mathrm{T}$ cell analysis in fresh blood as well as in cryopreserved PBMCs. Whole blood from 6 healthy individuals was collected before (day 0 ) and 7, 10, and 20 days after the prime dose and then 7, 10, 20, and 30 days after the boost dose. Whole fresh blood (2-6 hours after collection) was either directly stimulated with peptides for a cytokine release assay (CRA) or processed by Ficoll density gradient centrifugation to obtain isolated PBMCs (Figure 1A). PBMCs were either used fresh in the IFN- $\gamma$ ELISPOT assay (Figure 1A) or cryopreserved for further analysis. Fresh blood and fresh PBMCs were stimulated with the SpG peptide pool containing fifty-five 15 mer peptides (Supplemental Table 1; supplemental material available online with this article; https:// doi.org/10.1172/JCI152379DS1) covering spike-specific T cell epitopes that are immunogenic in $95 \%$ of SARS-CoV-2-infected individuals (5). Two negative controls consisting of the vehicle control with an identical DMSO concentration present in the SpG peptide pool and a peptide pool covering SARS-CoV-2 nucleoprotein (NP) (41 peptides covering the C-terminal half of the NP; Supplemental Table 1) were used. We measured IFN- $\gamma$ and IL-2 levels in the whole blood after 14-18 hours of incubation and enumerated the spots by ELISPOT assay following overnight incubation.

Figure $1 \mathrm{~B}$ shows that the 2 different assays detected a predominant spike-specific response in all the individuals and defined a matching profile of spike-specific $\mathrm{T}$ cell responses after the prime and boost vaccinations. The number of IFN- $\gamma$ spots detected after boost vaccination matched that observed in the phase I/II trial involving individuals vaccinated with BNT162b2 (10) and with a similar preparation consisting of the trimerized secreted version of the spike receptor-binding domain (BNT162b1; ref. 13), including a trial conducted in Chinese individuals vaccinated with BNT162b1 (21). In our study, although stimulation with the NP-specific peptide pool remained largely negative, the levels of IFN- $\gamma$ in the blood and the number of IFN- $\gamma$ spots showed identical peak responses that occurred 7-10 days after the first dose in individuals V4 and V5, and 7-10 days after the second dose in individuals V1, V3, and V6. There were, however, some minor discrepancies. The CRA did not detect boosting of spike-specific $\mathrm{T}$ cells induced by the second vaccine dose in subjects V4 and $\mathrm{V} 5$, perhaps in relation to the transient lymphopenia induced by the mRNA vaccination (13). IL-2 cytokine measurement (Figure 1C) revealed a pattern of spike-specific $\mathrm{T}$ cell responses equivalent to that achieved through IFN- $\gamma$ release. However, IL-2 levels exceeded those of IFN- $\gamma$ in all individuals 21 days after the first and second vaccine doses. Overall, we found a very strong correlation between IL-2 and IFN- $\gamma$ secretion and the number of IFN- $\gamma$ spots (Figure 1D), which allowed a precise estimation of the quantity of IFN- $\gamma$-producing cells related to the quantity of cytokines detected in whole blood (Table 1).

Assessment of the spike-specific $T$ cell response directly from fresh whole blood yields results comparable to those obtained with classical $T$ cell assays. Since $\mathrm{T}$ cell analysis is often performed in a single centralized laboratory using cryopreserved samples collected at different sites, we also analyzed the spike-specific $\mathrm{T}$ cell response after vaccination by performing ELISPOT activation-induced cellular marker (AIM) assays using cryopreserved samples stimulated with an SpG peptide pool. We then compared the results with those from the ELISPOT and CRA performed using the corresponding fresh whole blood. As already shown (22), the quantity of spike-specific spots detected by ELISPOT in cryopreserved PBMCs was reduced in comparison with the quantity detected in freshly isolated PBMCs (Supplemental Figure 1A), but the dynamics of the spike-specific response remained consistent with fresh 
A

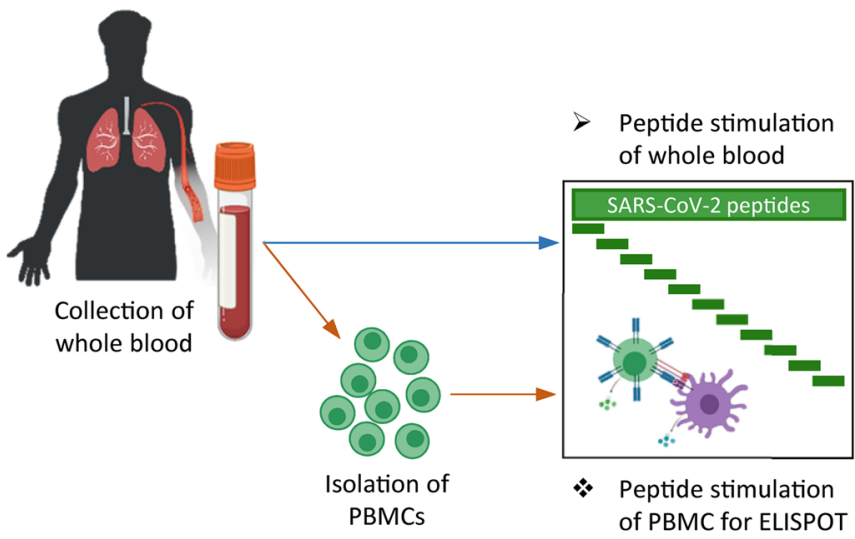

B

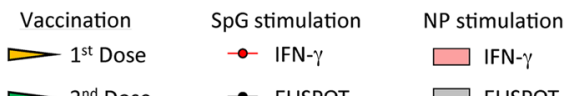

Plasma collection and analysis of secreted cytokines

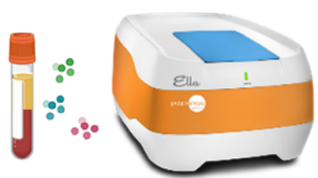

Work time : 3 hours Results in $<24$ hours

\section{Overnight} incubation

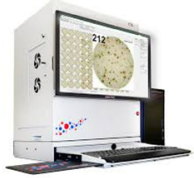

Work time : 10 hours Results in 36 hours

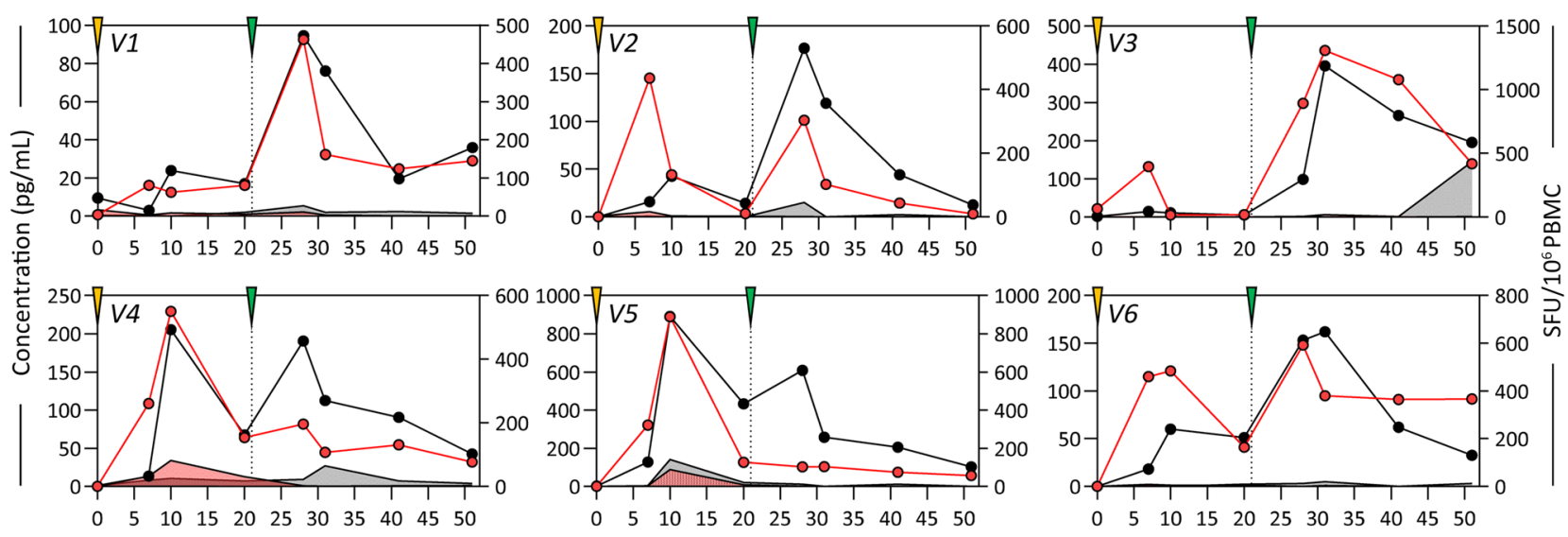

C
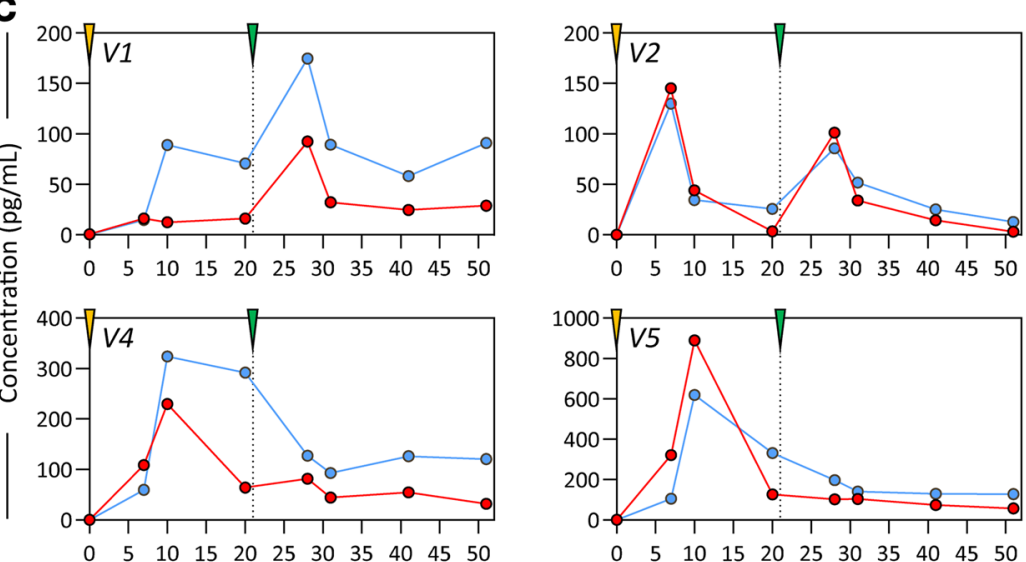

ELISPOT assay development and data acquisition
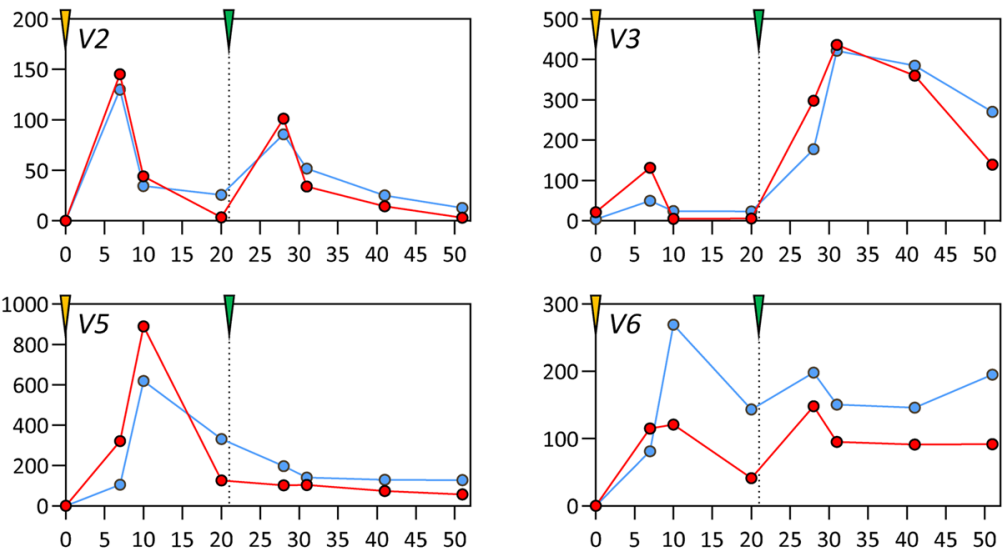

SpG stimulation

$\rightarrow$ IFN- $\gamma$

$\rightarrow$ IL-2
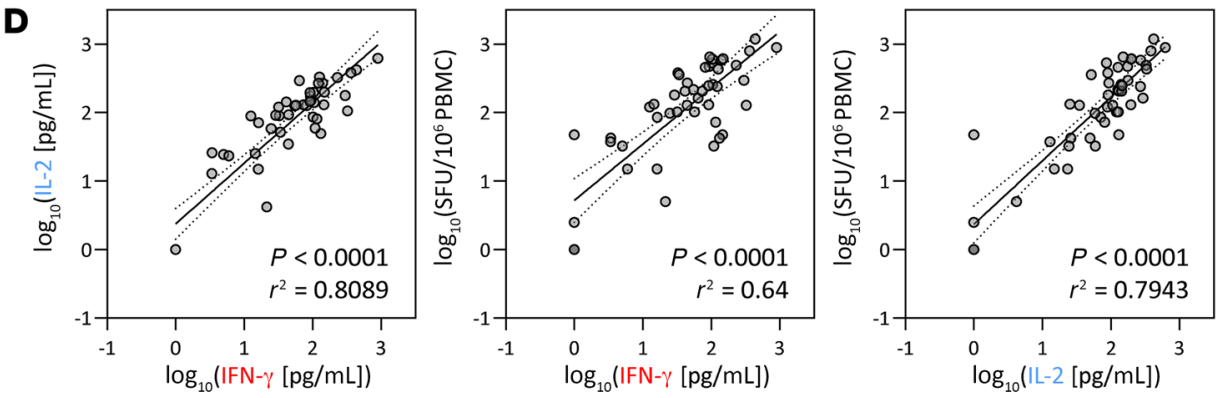
Figure 1. Detection of SARS-CoV-2 spike-specific T cells by peptide stimulation of whole peripheral blood from vaccinated individuals. (A) Schematic representation of the workflow for the direct peptide stimulation of whole peripheral blood and the subsequent detection of cytokine secretion compared with a standard IFN- $\gamma$ ELISPOT assay. (B) Six healthy individuals were vaccinated with 2 doses of BNT162b2 according to the recommended schedule ( 21 days apart), and whole-blood samples were longitudinally analyzed $7,10,20$, and 30 days after each dose. The collected whole blood was either directly stimulated for 16 hours with peptide pools specific for the spike protein (red or black line) or NP (red or black shaded area), or immediately processed with Ficoll density gradient centrifugation to isolate PBMCs. A standard IFN- $\gamma$ ELISPOT assay using the SpG- or NP-specific peptide pools was then set up using the freshly isolated PBMCs. The quantity of secreted IFN $-\gamma$ in stimulated whole blood (red line) was compared with the frequency of peptide-reactive PBMCs quantified by IFN- $\gamma$ ELISPOT (black line). (C) The levels of secreted IL-2 (blue line) in whole blood stimulated with the SpG peptide pool were compared with the amount of IFN- $\gamma$ detected. (D) Linear regression analysis of the concentrations of IFN- $\gamma$ and IL-2 in SpG-specific peptide poolstimulated whole blood and the corresponding frequency of spike-specific PBMCs ( $n=6 ; 48$ samples). Dotted lines denote the $95 \% \mathrm{Cl}$.

PBMCs (Supplemental Figure 1A) as also evidenced by the high correlation between the ELISPOT results from the differently processed samples (Figure 2A and Supplemental Figure 1B). The AIM assay, in our case, was less precise at detecting the dynamic expansion and contraction pattern of the spike-specific $\mathrm{T}$ cell response (Supplemental Figure 1, C and D), probably because of the negative effect of cryopreserving PBMCs. Nevertheless, the ability of the AIM assay to differentiate between $\mathrm{CD} 4^{+}$and $\mathrm{CD}^{+} \mathrm{T}$ cell responses is an asset that should not be discounted.

We then assessed whether whole-blood CRA results could reflect those obtained using other assays (Figure 2, A and B). We correlated the results obtained in all the different assays of spike-specific T cells in cryopreserved and fresh PBMC samples with the results from the whole-blood CRA. We found that cytokines in whole blood remained well correlated with ELISPOT assays in which cryopreserved or fresh PBMC samples were used,

\section{Table 1. Estimated IFN $-\gamma$ SFU/10 6 PBMCs derived from IFN $-\gamma$ and IL-2 concentrations in SpG peptide pool-stimulated whole blood, based on the linear regression analysis in Figure 10}

IFN- $\gamma /$ IL-2 concentration SFU $/ 10^{6}$ PBMCs estimated SFU $/ 10^{6}$ PBMCs estimated $(\mathrm{pg} / \mathrm{mL}) \quad$ from IFN- $\gamma$ concentration from IL-2 concentration

\begin{tabular}{|ccc|}
\hline 1 & 5.2 & 2.3 \\
\hline 10 & 34.6 & 19.8 \\
\hline 25 & 73.6 & 46.4 \\
\hline 50 & 130.1 & 88.3 \\
\hline 75 & 181.7 & 128.7 \\
\hline 100 & 230.2 & 168.2 \\
\hline 200 & 407.1 & 320.3 \\
\hline 300 & 568.3 & 466.9 \\
\hline 400 & 720.0 & 609.9 \\
\hline 700 & 865.1 & 750.5 \\
\hline 1000 & 1207.7 & 1093.9 \\
\hline
\end{tabular}

while its correlation with the AIM assay results was generally weaker (Figure 2, A and B). These results indicate that the CRA, which utilizes freshly collected whole blood, is a robust method for reliable quantification of spike-specific $\mathrm{T}$ cell responses, producing results that are comparable to those obtained with well-established assays used to analyze $\mathrm{T}$ cell responses.

Determining the fine specificity of spike-specific $T$ cell responses through whole-blood CRA. We next tested whether the whole-blood CRA could be used to rapidly define $\mathrm{T}$ cell immunogenic regions of the whole spike protein in vaccinated individuals. We organized 15 mer peptides with an overlap of 10 aa covering the whole 1273 aa long spike protein into distinct pools of approximately 40 peptides that covered the following 7 spike regions: pool 1 (1-180 aa), pool 2 (171-345 aa), pool 3 (336-510 aa), pool 4 (501-705 aa), pool 5 (696-895 aa), pool 6 (886-1085 aa), and pool 7 (1076-1273 aa). A schematic representation of the localization of peptide pools 1-7 in relation to the $\mathrm{S} 1$ (N-terminal), $\mathrm{RBD}$, and $\mathrm{S} 2$ (C-terminal) regions of the spike protein is shown in Figure 3A. These different spike peptide pools were used in the whole-blood CRA and ELISPOT with freshly isolated PBMCs. The results of these different assays performed at the indicated time points are first shown in 2 representative vaccine recipients (Figure 3B), whereas the results obtained in all 6 individuals are represented as a heatmap in Figure 3C. The 3 different measurements (IFN- $\gamma$ and IL-2 CRA and IFN- $\gamma$ ELISPOT) provided very similar information in relation to the $\mathrm{T}$ cell response induced by BNT162b2 in healthy individuals. Even though some differences can be noted, like in individual $\mathrm{V} 3$, in whom the dominant IFN- $\gamma$ response (CRA and ELISPOT) was induced by pool 3, while pool 7 induced the dominant IL-2 response, overall, all the assays were largely equivalent. Consistent across the 3 different measurements, spike-specific T cells preferentially targeted the $\mathrm{S} 2$ chain of spike (covered by pools 5,6 , and 7 spanning spike 700-1273 aa), with responses in all 6 of the individuals tested at different time points. The whole-blood CRA and ELISPOT also showed that the region 501-705 aa contained in pool 4 was the least immunogenic, with only 1 of the 6 vaccine recipients showing a response at different time points (Figure 3, B and $\mathrm{C}$ ). Interestingly, analysis of individuals who recovered from SARS-CoV-2 infection (23) and of recipients of the mRNA vaccine (24) showed a similarly reduced frequency of responses in the spike region 500-700 aa for $\mathrm{CD}^{+} \mathrm{T}$ cells detected by AIM assay. Taken together, these data show that direct analysis of cytokines secreted in whole blood pulsed with different peptides constitutes a reliable method to gauge the presence and magnitude of functional T cells specific for epitopes covered by the peptides used.

A total spike-specific $T$ cell response is accurately represented by the T cells specific for the SpG peptide pool. Although the wholeblood CRA using the 7 overlapping peptide pools of the spike protein could provide us information on the immunogenicity of the different regions of spike and the total spike-specific $\mathrm{T}$ cell response, assessment of the response in larger numbers of individuals requires a more streamlined approach. Thus, we analyzed the relation between the total spike-specific $\mathrm{T}$ cell response and the response to our selected SpG peptide pool. A schematic representation of the localization of the peptides contained in the SpG peptide pool is shown in Figure 4A. By correlating the results from 3 different assays (IFN- $\gamma$ and IL-2 CRA and IFN- $\gamma$ 
A
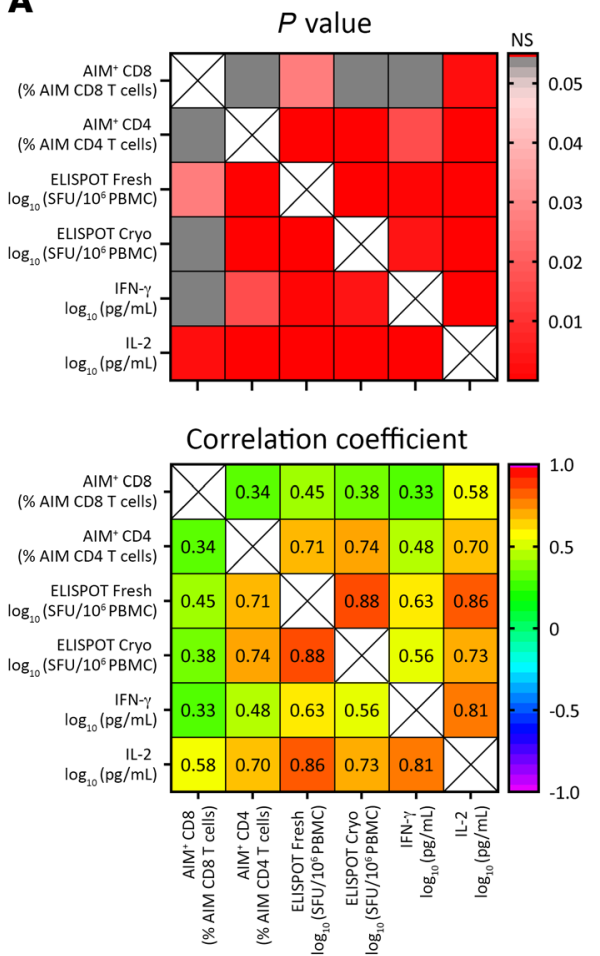

B

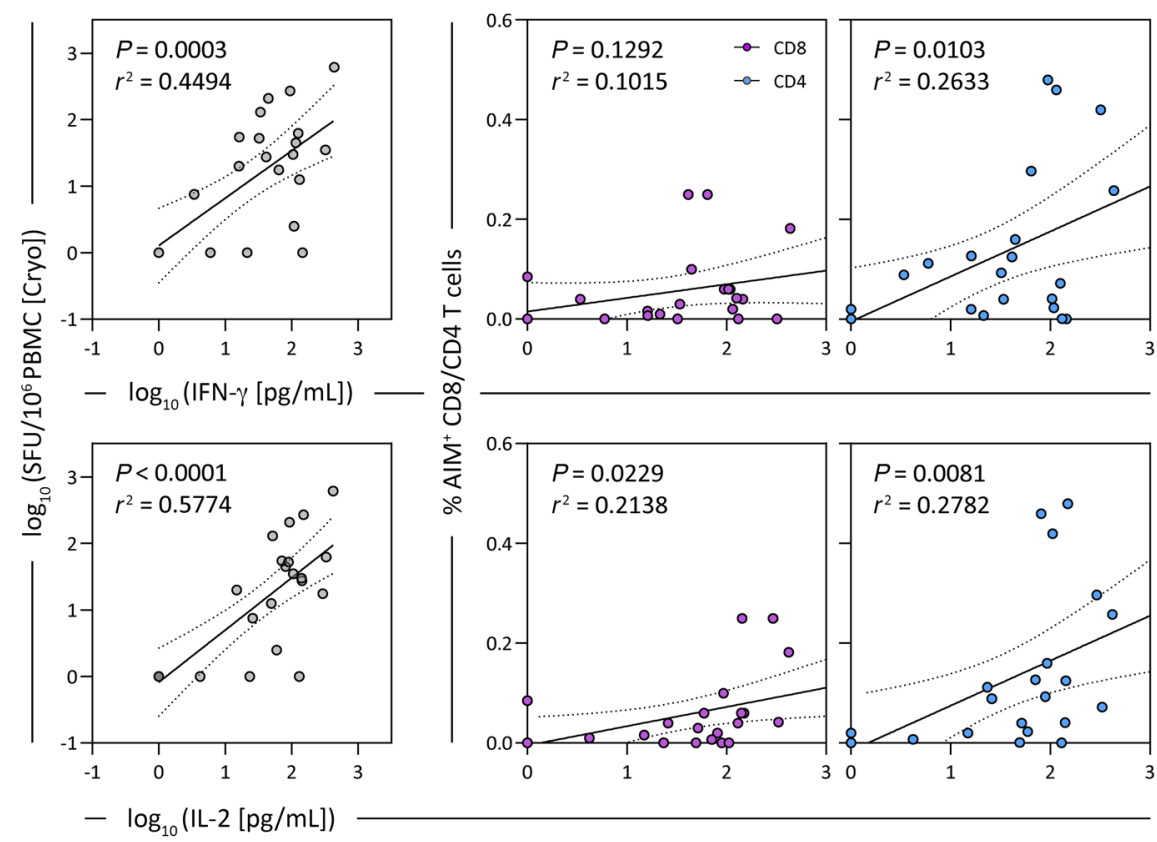

Figure 2. Correlation matrix of different assays used to quantify spike-specific $\mathbf{T}$ cells. (A) The top matrix shows the significance of the correlation, and the Spearman's correlation coefficient is shown in the matrix below ( $n=6 ; 24$ samples). (B) Linear regression analysis of the concentrations of IFN- $\gamma$ and IL-2 in SpG peptide pool-stimulated whole blood and the corresponding frequency of SpG-reactive T cells in cryopreserved PBMCs quantified by either IFN- $\gamma$ ELISPOT or AIM assay ( $n=6$; 24 samples). Dotted lines denote the $95 \% \mathrm{Cl}$.

ELISPOT), in which both the total spike protein and SpG peptide pool-specific $\mathrm{T}$ cell responses were determined in the same sample through stimulation with the corresponding peptide pools, we observed a strong positive linear relationship indicating that the $\mathrm{T}$ cell response against the SpG peptide pool was highly representative of the total spike $\mathrm{T}$ cell response (Figure 4B). In fact, the SpG peptide pool-specific $\mathrm{T}$ cell response constituted approximately $60 \%-80 \%$ of the total $\mathrm{T}$ cell response against the entire spike protein (Figure 4C). This was further supported by a linear regression analysis that also showed a good correlation between the IFN- $\gamma$ and IL-2 CRA data obtained through individual spike pool stimulation (pools 1-7) and total spike protein IFN- $\gamma$ ELISPOT data (Supplemental Figure 3). Hence, using the whole-blood CRA with SpG peptide pool as a stimulant, we proceeded to analyze the spike-specific T cell response in a larger cohort of BNT162b2vaccinated individuals and of individuals who had recovered from SARS-CoV-2 and SARS-CoV-1 infection.

$T$ cell responses to spike after vaccination or after natural infection with SARS-CoV-2 or SARS-CoV-1. A total of 112 individuals vaccinated with BNT162b2 (201 samples), 62 and 68 individuals who recovered from symptomatic (115 samples) and asymptomatic (62 samples) SARS-CoV-2 infection, respectively, and 12 individuals who recovered from SARS-CoV-1 infection 18 years ago (12 samples) were studied longitudinally using the whole-blood CRA with the SpG peptide pool and measuring both IFN- $\gamma$ and IL-2.

First, we analyzed samples collected 3 months or more after the boost vaccination ( 3 months) or SARS-CoV-2 infection clear- ance (3-12 months) to understand whether the whole blood CRA remained reliable for quantification of the spike-specific $\mathrm{T}$ cell responses at later time points beyond that tracked in Figures 1 and 5. Linear regression analysis of IFN- $\gamma$ and IL-2 secretion in a whole-blood CRA and the corresponding frequency of IFN- $\gamma$ spots in the ELISPOT assay showed a good correlation across all groups at time points of 3 months and beyond (Figure 5). This finding indicated that the whole-blood CRA can quantify spike-specific $\mathrm{T}$ cell responses accurately in vaccinated and infected individuals within 3-12 months of a boost vaccination or infection resolution, respectively.

We analyzed all the time points studied and found that vaccinated individuals had a pronounced spike-specific $\mathrm{T}$ cell response at the earliest analyzed time point ( 14 days) after the boost vaccination dose, which gradually declined and started to stabilize above the positivity threshold at 2-3 months (34 of 35 individuals were IFN- $\gamma$ positive at 3 months), consistent with an initial $\mathrm{T}$ cell clonal expansion induced by vaccination and subsequent normalization (Figure 6A and Supplemental Figure 4). We observed similar kinetics in individuals with natural SARS-CoV-2 infection, in whom spike-specific $\mathrm{T}$ cell responses were high approximately 1 month after infection clearance and gradually declined and stabilized above the positivity threshold (asymptomatic: 23 of 27 asymptomatic individuals were IFN- $\gamma$ positive at 9-12 months; symptomatic: 46 of 55 were IFN- $\gamma$ positive at 9-12 months), regardless of the symptomatic presentation during infection (Figure 6A and Supplemental Figure 4). Even in individuals who recovered 
A

Spike overlapping peptide pools
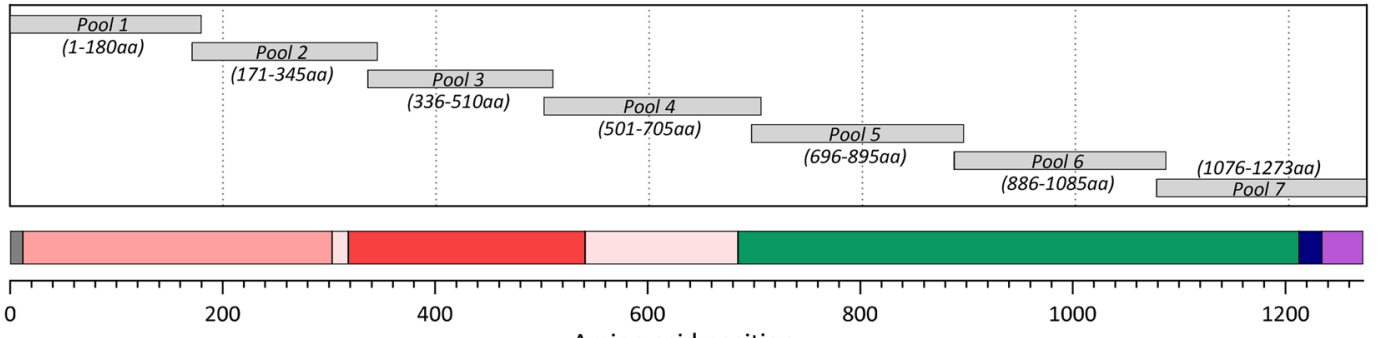

Amino acid position

B

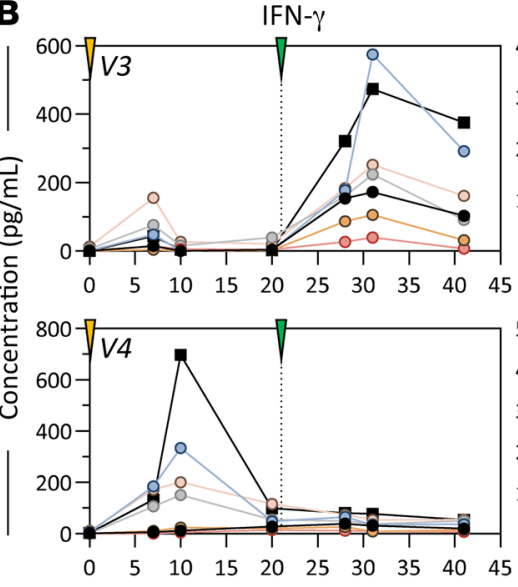

IL-2
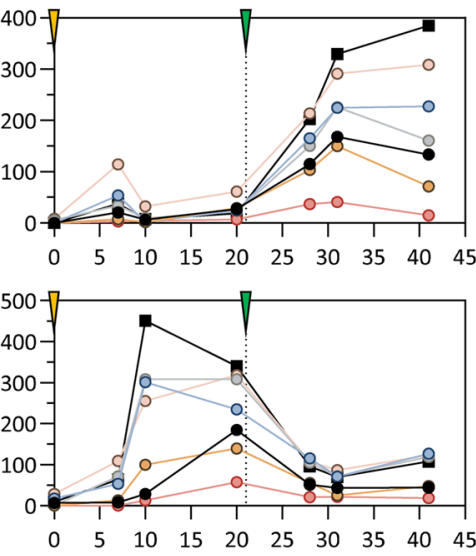

IFN- $\gamma$ ELISPOT

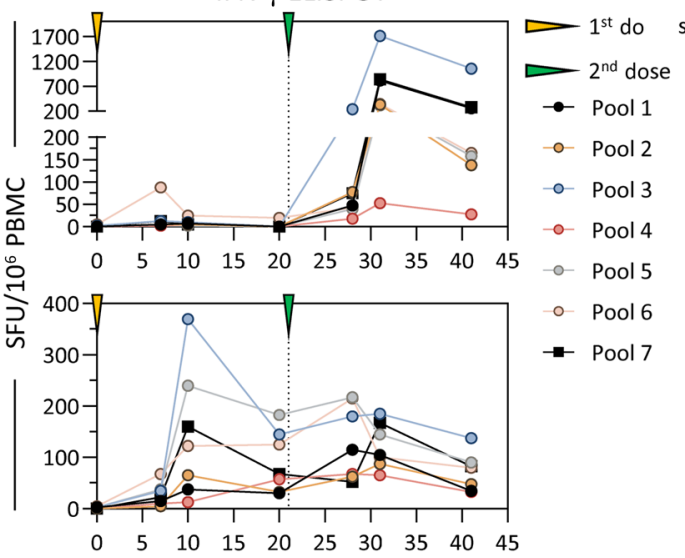

C
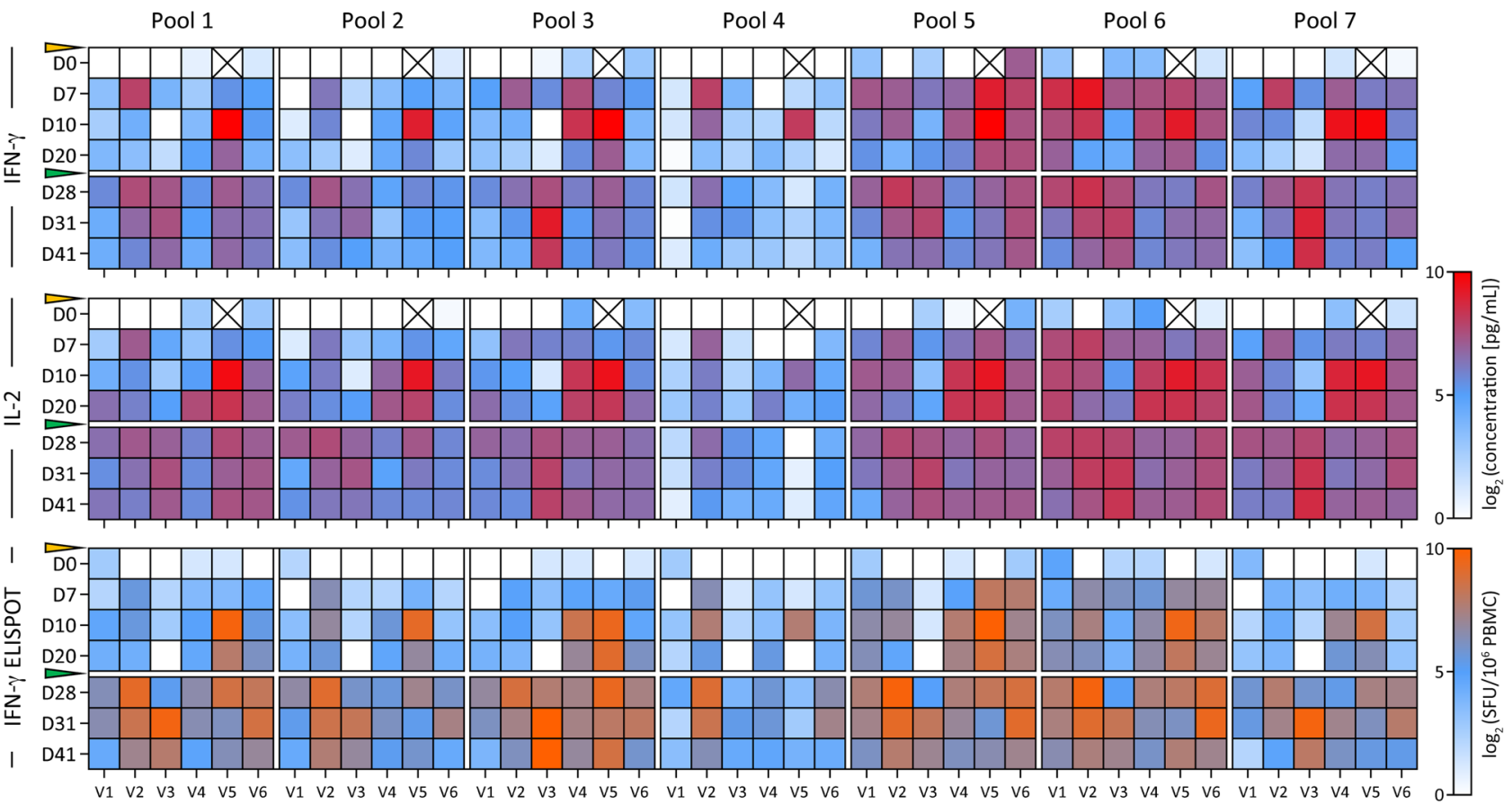

Figure 3. Immunodominance of spike-specific T cells in vaccinated individuals. (A) Schematic representation of the 7 spike-specific peptide pools containing 15 mer overlapping peptides spanning the entire spike protein. Pools 1-4 contain peptides from the signal peptide and the S1 chain, whereas pools 5 and 6 encompass the S2 chain together with the transmembrane and cytoplasmic domains. (B) Plots show the longitudinal evaluation of spike-specific T cell responses (pools 1-7) by quantification of IFN- $\gamma$ (left) or IL-2 (middle) in peptide-stimulated whole blood, or by IFN- $\gamma$ ELISPOT (right) in 2 representative vaccinees. (C) Heatmap shows the spike-specific T cell responses quantified longitudinally in all vaccinees $(n=6)$ using the 3 different assays described above. " $X$ " denotes time points that were untested. 
A SpG overlapping peptide pools (55 peptides)
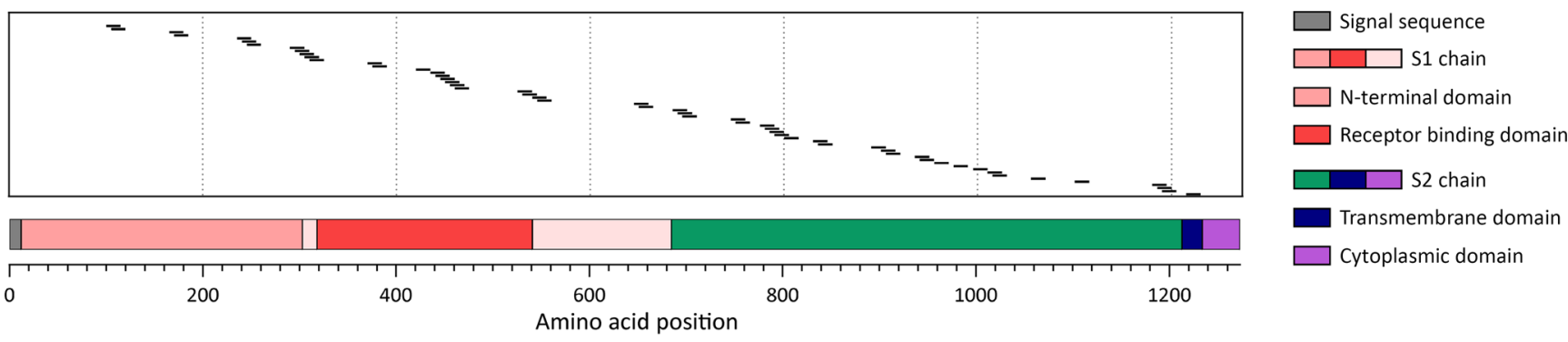

B

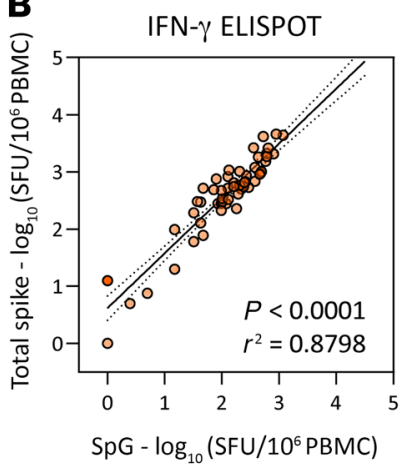

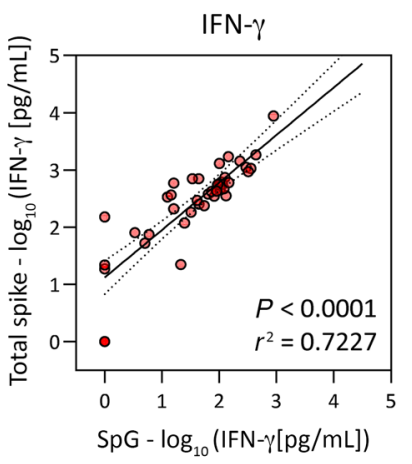

C

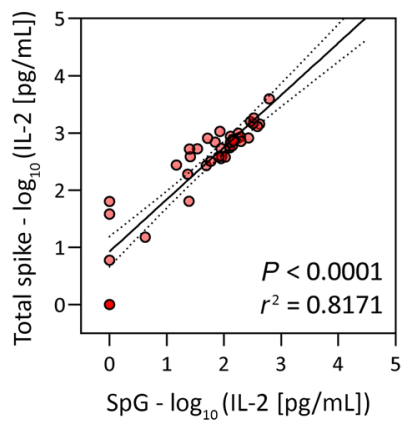

SpG response

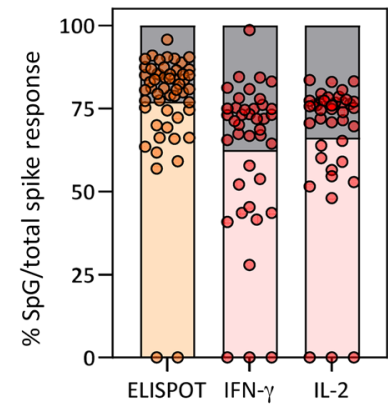

Figure 4. Frequency of the SpG peptide pool and total spike protein-specific T cells. (A) Schematic representation of the individual 15 mer overlapping peptides contained in the SpG peptide pool. (B) Linear regression analysis of the T cell response against the SpG peptide pool and the total spike protein (pools 1-7) as evaluated by ELISPOT (left) or by the quantification of IFN- $\gamma$ (middle) or IL-2 (right) in peptide-stimulated whole blood ( $n=6 ; 42$ samples). (c) The SpC peptide pool-specific T cell response quantified by each assay is expressed as a fraction of the total spike protein T cell response observed ( $n=6 ; 42$ samples). Bars indicate the mean.

from SARS-CoV-1 infection 17 years ago, T cells specific for SARSCoV-2 spike protein also remained detectable (8 of 12 were IFN- $\gamma$ positive), similar to the NP-specific $\mathrm{T}$ cell responses described previously (25), despite the low aa conservation of the SpG peptides between the 2 viruses (Figure 6A). Clearly, further analysis is needed to determine whether $\mathrm{T}$ cells induced by vaccines will be maintained at levels similar to those induced by natural infection beyond the 3-month period.

We also assessed whether there were differences in the magnitude of the responses between the different groups. We compared the spike-specific $\mathrm{T}$ cell responses detected at similar time points 2-3 months after boost vaccination or viral clearance. Unlike the reports of higher neutralizing antibody titers in vaccinees (13), we found that individuals with symptomatic SARS-CoV-2 infection and vaccinees mounted equivalent magnitudes of spike-specific T cell responses (both IFN- $\gamma$ and IL-2 secretion), whereas higher levels of IFN- $\gamma$ secretion were only detected in individuals who had an asymptomatic SARS-CoV-2 infection (Figure 6B). The latter observation is in line with previous analyses of asymptomatic and symptomatic SARS-CoV-2infected individuals within 1 month of viral clearance, in which the former showed increased cytokine production with comparable frequencies of virus-specific $\mathrm{T}$ cells (5). Subtle qualitative differences in the spike-specific $\mathrm{T}$ cell response were also observed in the different groups. Upon vaccination, spike peptide-induced IFN- $\gamma$ and IL-2 secretion levels were comparable, but gradually diverged over time, leading to higher levels of detectable IL-2 two to 3 months after boost vaccination (Figure
6C). Symptomatic individuals also produced significantly more IL- 2 than IFN- $\gamma$ more than 6 months after viral clearance, while this difference was less pronounced in asymptomatic individuals even at the latest time points tested (9-12 months after viral clearance), which did not reach statistical significance (Figure 6C). In individuals with previous SARS-CoV-1 infection, wholeblood CRA also detected higher IL-2-secreting, spike-specific $\mathrm{T}$ cell responses 18 years after resolution of infection (Figure 6A). Hence, quantification of IL-2 secretion provides better sensitivity than IFN- $\gamma$ in identifying individuals with a long-term spike-specific memory $\mathrm{T}$ cell response.

Whole-blood CRA detects the wide dynamic range and heterogeneous function of spike-specific $T$ cell responses in vaccinated individuals. In addition to evaluating the kinetics as well as quantitative and qualitative differences in $\mathrm{T}$ cell responses, the whole-blood CRA also detected a wide range of spike-specific $\mathrm{T}$ cell responses in vaccinated individuals. Figure 7A shows the paired longitudinal samples of 27 vaccinees approximately 14 and 90 days after boost vaccination. The levels of secreted IFN- $\gamma$ and IL- 2 in the wholeblood CRA differed between the 2 time points and among individuals. Interestingly, the quantity of cytokines detected 2 weeks after boost-dose vaccination did not always predict the level of spike-specific $\mathrm{T}$ cell responses measurable by day 90 . Some individuals had a greater than 20-fold reduction in IFN- $\gamma$ and IL-2 levels on day 90 after boost vaccination, while in others, the levels, particularly those for IL-2, were more stable (Figure 7A). Indeed, some individuals have a more pronounced decline in IFN- $\gamma$ levels than IL-2 levels, or vice versa, as indicated in Figure 7B, in which 
$\geq 3$ mos. after boost/viral clearance

Vaccinated ( $n=30 ; 30$ samples)
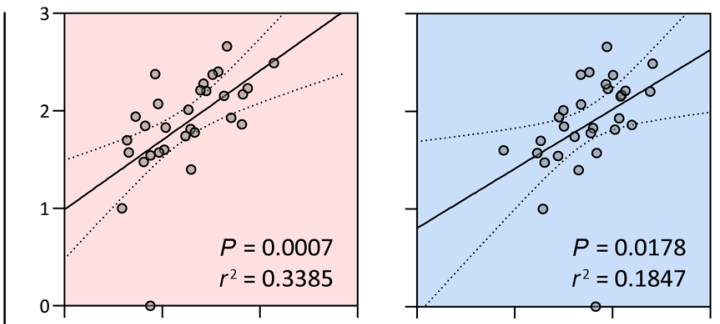

Asymp. COVID-19 ( $n=51 ; 51$ samples)
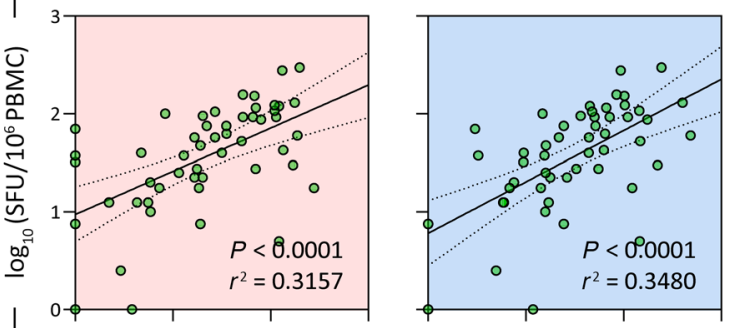

Symp. COVID-19 ( $n=38 ; 62$ samples)
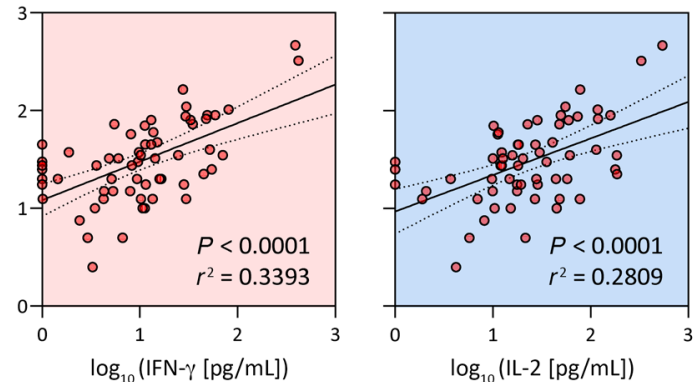

Figure 5. Whole-blood CRA quantifies spike-specific memory $T$ cell responses $\mathbf{3}$ or more months after the boost vaccination dose or viral clearance. Linear regression analysis of IFN- $\gamma$ and IL-2 concentrations in SpC peptide pool-stimulated whole blood and the corresponding frequency of spike-specific T cells quantified by IFN- $\gamma$ ELISPOT in vaccinated individuals (gray; $n=30$; 30 samples) and in asymptomatic (Asymp., green; $n=51$; 51 samples) and symptomatic (Symp., red; $n=38 ; 62$ samples) COVID-19 patients sampled 3 months or more after the boost vaccination dose or viral clearance. Dotted lines indicate the $95 \% \mathrm{CI}$. SFU, spot-forming units.

the trajectory of IFN- $\gamma$ and IL-2 levels on day 14 and day 90 in each individual is plotted.

Spike-specific $T$ cell responses do not correlate with neutralizing antibody quantities. Last, since the quantification of serum neutralizing antibodies is the mainstay of SARS-CoV-2 humoral immunity assessment, we determined whether there was a predictive relationship between the quantity of neutralizing antibodies and spike-specific $T$ cell responses. We performed linear regression analysis of the $\mathrm{T}$ cell response in vaccinated and convalescent asymptomatic and symptomatic COVID-19 patients, quantified through whole-blood CRA (both IFN- $\gamma$ and IL-2) with assessment of serum neutralizing antibody quantities through an RBD-hACE2 binding inhibition assay (Figure 8). We found no substantial correlations in any of the analyzed groups. As also demonstrated previously in convalescent $\{\mathrm{PhD}: 2021 \mathrm{bm}\}$ patients and in vaccinated individuals (26), serum neutralizing antibody quantities cannot predict the corresponding spike-specific $\mathrm{T}$ cell responses in an individual, and this further stresses how $\mathrm{T}$ cell response information from the whole-blood CRA complements existing antibody assessments.

\section{Discussion}

The complexity of virus-specific $\mathrm{T}$ cell characterization relegates their analysis to studies performed in selected laboratories experienced in the complex methods of $\mathrm{T}$ cell analysis. The rapidity, simplicity, and accuracy of the CRA in whole blood can allow for routine measurement of SARS-CoV-2 $\mathrm{T}$ cells in large populations and thus further our understanding of the role of antiviral $\mathrm{T}$ cells during the current COVID-19 pandemic. It is important to note that measurement of cytokine release in stimulated whole blood not only detects the mere presence of $\mathrm{T}$ cells but determines their functionality as well. This is an important feature that differentiates the CRA (and other assays like ELISPOT and AIM) from the recently developed T-Detect COVID test (Adaptive Biotechnologies), which uses next-generation sequencing of $\mathrm{T}$ cell receptors (TCRs) to determine the presence or absence of cellular immunity to SARS-CoV-2 (27). At the same time, although a functional readout is of benefit, the whole-blood CRA, like ELISPOT, does not differentiate between functional $\mathrm{CD} 4^{+}$and $\mathrm{CD} 8^{+} \mathrm{T}$ cells. Hence, such an approach is not suitable for determining the quantities or unique functionality of different $\mathrm{T}$ cell subsets. The use of peptides to stimulate and detect specific $\mathrm{T}$ cells could also bias the detection toward high-avidity $\mathrm{T}$ cell responses. However, this is not unique to the whole-blood CRA, as other assays like ELISPOT, intracellular cytokine staining (ICS), and AIM also use isolated PBMCs stimulated with similar quantities of peptide. Nonetheless, we think that the ability of the whole-blood CRA to measure the wide, dynamic range of functional spike-specific $\mathrm{T}$ cells and not just their presence will be an important asset that will more precisely evaluate the protective ability of $\mathrm{T}$ cells after infection or vaccination.

In this study, by sequentially testing vaccinated and SARSCoV-2-convalescent individuals, we show that IL-2 and IFN- $\gamma$ quantification in whole blood measured spike-specific $\mathrm{T}$ cell responses with an accuracy equivalent to that obtained with ELISPOT assays performed in freshly purified PBMCs. Minor discrepancies between the magnitudes of $\mathrm{T}$ cells were detected only at early time points when the CRA was able to detect a signal in the absence of ELISPOT results. Furthermore, analysis 2 and 3 months after vaccination showed, on average, a better sensitivity of IL-2 than IFN- $\gamma$ in detecting spike-specific T cell responses in individuals. The superior ability of IL-2 to detect long-term memory spike-specific $T$ cells was also supported by the analysis of SARSCoV-2-convalescent individuals 12 months after infection and also 17 years after infection in the case of SARS-CoV-1-infected individuals. Interestingly, this difference in sensitivity was less pronounced in convalescent COVID-19 patients with asymptomatic infection. Though speculative at the moment, this lack of difference between IFN- $\gamma$ and IL-2 secretion levels could reflect a better functionality of spike-specific $\mathrm{T}$ cell responses that would hence plausibly contribute to the benign disease trajectory for these individuals.

The ability of the CRA to measure the dynamic range of spike-specific $\mathrm{T}$ cell responses allowed us to study a wide group of 
A Vaccinated

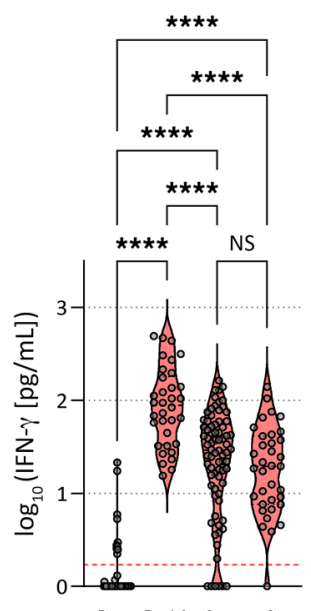

Pre $D^{\sim 14} 2 \mathrm{mo} 3 \mathrm{mo}$
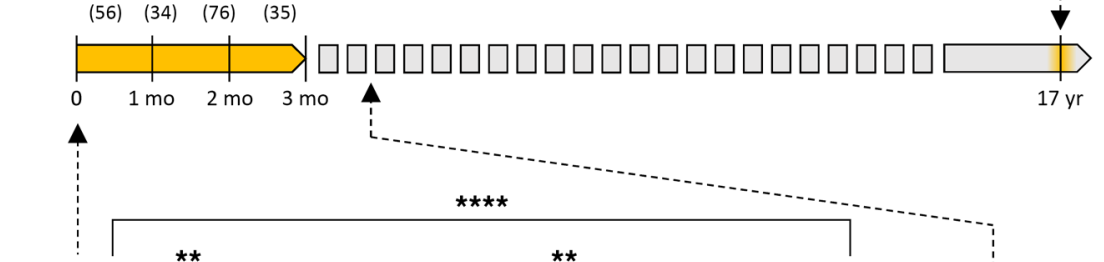
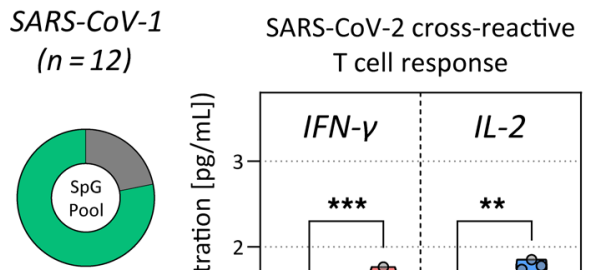

12 Conserved

(21.82\%)

43 Unique
$(78.18 \%)$

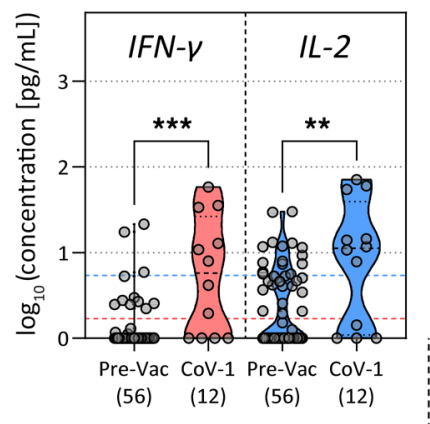

B

2-3 mo after boost/viral clearance
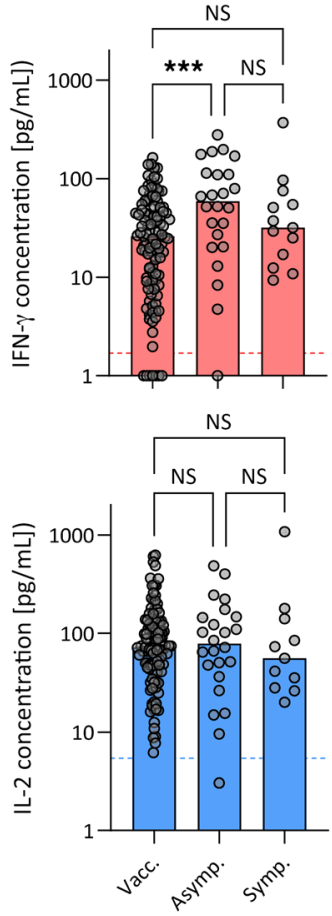

C -0 IFN- $\gamma \quad-\circ$ IL-2
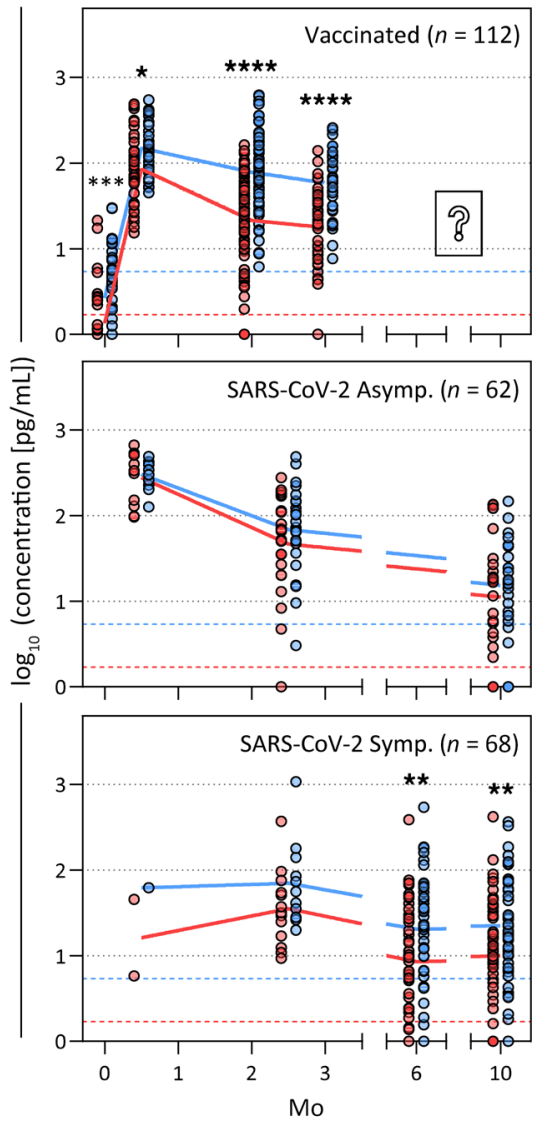
Figure 6. Longitudinal quantification of spike-specific $T$ cells from whole blood in vaccinated and infected individuals. (A) SARS-CoV-2 spikespecific T cell responses in vaccinated individuals ( $n=112$; 201 samples) and in convalescent asymptomatic ( $n=62 ; 62$ samples) and symptomatic ( $n=68$; 115 samples) COVID-19 patients were longitudinally quantified by measuring IFN- $\gamma$ secretion in whole blood after $\mathrm{SpC}$ peptide pool stimulation. Cross-reactive SARS-CoV-2 spike-specific T cells were also quantified in whole blood from individuals who were infected with SARSCoV-1 eighteen years ago ( $n=12 ; 12$ samples). The responses of individuals before receiving BNT162b2 vaccination are shown for reference. Pie chart shows the number of peptides in the SpC peptide pool that are conserved or unique between SARS-CoV-2 and SARS-CoV-1. The sampling timespan (highlighted in yellow) is shown, and the number of samples analyzed at each time point is indicated in parentheses. Dashed lines denote the detection cutoff for the measured cytokines. D 14, day 14. (B) Quantities of secreted IFN- $\gamma$ (red) and IL-2 (blue) in SpG peptide pool-stimulated whole blood from vaccinees and COVID-19 patients sampled 2-3 months after a boost vaccination dose (Vacc.) or viral clearance. The bars indicate the median value for each group, and the dashed lines indicate the detection cutoff for the measured cytokines. Significant differences were analyzed and are displayed as above. (C) Longitudinal dynamics of secreted IFN- $\gamma$ (red) and IL-2 (blue) in SpG peptide pool-stimulated whole blood from vaccinees and patients with COVID-19. Dashed lines indicate the detection cutoff for the measured cytokines. Significant differences in each group were analyzed by 1-way ANOVA, and the $P$ value (adjusted for multiple comparisons) are shown. NS $=P>0.05 .{ }^{*} P \leq 0.05,{ }^{*} P \leq 0.01$, ${ }^{* *} P \leq 0.001$, and ${ }^{* * * *} P \leq 0.0001$.

vaccinated and COVID-19-convalescent individuals. We found that, despite the homogeneous cohort of vaccinated adults (21-60 years of age, healthy and SARS-CoV-2-naive individuals), the spike-specific $\mathrm{T}$ cell response was quantitatively different immediately after both the prime and boost vaccination doses ( 14 days after vaccination) and after 3 months, similar to what was detected in a more heterogeneous population of COVID-19-convalescent individuals with mild or asymptomatic disease. In the vaccinated cohort, we first observed that half of the individuals showed a peak of their spike-specific $\mathrm{T}$ cell response approximately 14 days after the prime dose, whereas the other half reached their peak response after the boost dose. These data were derived from a very limited sample size, since we studied only 6 individuals at multiple time points after vaccination. However, the inability of the second vaccine dose to boost spike-specific $\mathrm{T}$ cell (and antibody) responses in some individuals has been observed so far only after vaccination of SARS-CoV-2 convalescents and not, like in our study, in SARS-CoV-2-naive individuals. It is possible that the preexisting spike-specific $\mathrm{T}$ cells primed by other coronaviruses influenced the different kinetics in this case, a phenomenon that was recently demonstrated in vaccinees receiving low doses of the mRNA-1273 vaccine (28).

Our analysis of a much larger cohort of vaccinated individuals ( $n=112)$ studied with less frequent sampling up to 3 months upon completion of vaccination further confirmed the heterogeneity of the magnitude of the induced spike-specific cellular response. The quantity and kinetics of decline of the spike-specific cellular responses diverged among the vaccinated individuals, with CRA IFN- $\gamma$ and IL-2 concentrations that ranged from $10-500 \mathrm{pg} / \mathrm{mL}$ and $40-500 \mathrm{pg} / \mathrm{mL}$, respectively. Interestingly, some individuals have a constant cytokine secretion level over the observation period, while in others, the cytokine quantities dropped precipitously. In addition, the longitudinal quantification of 2 cyto- kines further increased the heterogeneity of the vaccine-induced immunity in different individuals, as IFN- $\gamma$ and IL-2 quantities did not decrease in parallel in all the individuals. Some vaccinees displayed stable IL-2 production levels associated with a profound decrease in IFN- $\gamma$ levels, whereas others showed exactly the opposite. Whether these differences can be attributed to the presence of different populations of effector/memory $\mathrm{T}$ cells or different ratios of $\mathrm{CD}^{+}$and $\mathrm{CD} 8^{+} \mathrm{T}$ cells, and whether such differences have an impact on protection will need to be analyzed in a large clinical study. In addition, it will be important to continue monitoring the spike-specific $\mathrm{T}$ cell response beyond the 3-month observation period to understand whether the spike-specific $\mathrm{T}$ cell response induced by vaccines will behave like the one observed after natural infection. Importantly, the rapid cytokine assay was able to detect spike-specific T cells in approximately $84 \%$ of individuals 1 year after infection with SARS-CoV-2 and also in 8 of 12 individuals 18 years after SARS-CoV-1 infection.

Another important observation that further supports the concept of marked heterogeneity of the immune response induced by the BNT162b2 vaccine was the lack of correlation between the magnitudes of humoral and cellular immunity. The substantial independence of different components of the immune system after the initial induction phase has been demonstrated in COVID-19 convalescents $(29,30)$ and can be explained by recent data showing that neutralizing antibodies can be produced without follicular T cell help (31). The CRA also revealed similar profiles in both COVID-19-convalescent and vaccinated individuals, as no correlation was detected between the neutralizing antibody titers and the magnitude of cytokine secretion in these individuals. COVID-19 convalescents were studied more than 1 year after infection and had different levels of neutralizing antibodies, whereas vaccinated individuals mostly had consistently high neutralizing antibody titers with little variation among those tested 3 months after vaccination. In other studies, antibody persistence was still observed up to 6 months after mRNA vaccination at the time of the report (32). We do not know whether antibodies induced by vaccination will show a rate of decline similar to that observed after natural infection beyond the 6-month follow-up date, which makes the analysis of cellular immunity even more important. Our data at the moment show that 3 months after vaccination, the levels of neutralizing antibodies cannot be used as a surrogate for the spikespecific cellular immunity induced by vaccination.

This quantitative heterogeneity was, however, not mirrored by the regions of spike protein targeted by $\mathrm{T}$ cells induced by BNT162b2 vaccination. We observed a substantial similarity of immunodominance among the different vaccinated individuals, with a large part of the $\mathrm{T}$ cell response directed toward the spike 2 chain and with an almost complete lack of $\mathrm{T}$ cell determinants within the N-terminal region of S1 (region 501-705 aa). A reduced presence of $\mathrm{T}$ cell epitopes in this region was already observed in SARS-CoV-2 convalescents (23). It will be interesting to test whether this documented profile of spike-T cell specificity will also occur in individuals vaccinated with different products, where subtle differences in codon usage, signal peptides, and aa modifications (pre-fusion conformation stabilization and furin cleavage site mutations) were introduced (33-36). Of note, an intermediate dominance of the RBD-containing spike pool 3 was also observed, 


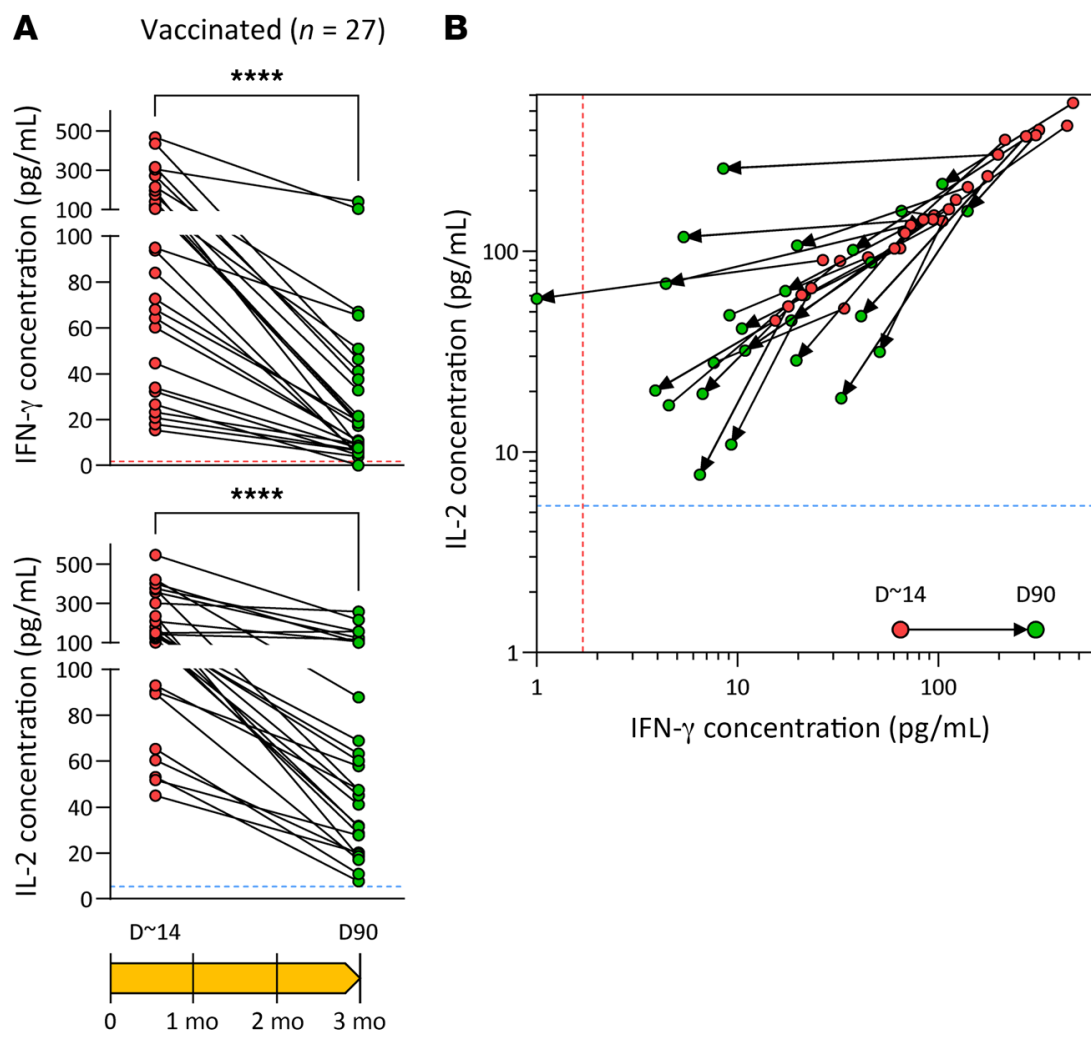

Figure 7. Heterogeneity of spike-specific $T$ cell responses in vaccinated individuals. (A) SARSCoV-2 spike-specific T cell responses were evaluated by SpC peptide pool stimulation of whole blood from vaccinated individuals $(n=27) 2$ weeks (green circle) and 3 months (red circle) after the boost vaccination dose. The secreted IFN- $\gamma$ and IL-2 concentrations are shown. ${ }^{* * *} P<\leq$ 0.0001 , by Wilcoxon matched-pairs signed-rank test. (B) Bivariate dot plots of secreted IFN- $\gamma$ and IL-2 concentrations. Arrows connect paired individuals analyzed on day 14 and day 90 . Dashed lines indicate the detection cutoff for the measured cytokines.

were quantified using cPASS (GenScript), and all were negative.

Peptides. Peptides of 15 mer that overlapped by 10 aa spanning the entire SARS-CoV-2 spike protein (GISAID EPI_ISL_410713) were synthesized (GenScript) and pooled into 7 pools of approximately 40 peptides in each pool (Supplemental Table 1). Fifty-five spike peptides covering the immunogenic regions of the SARS-CoV-2 spike protein that represent $40.5 \%$ of the whole spike protein formed the SpG peptide pool as described previously (5).

CRA of whole peripheral blood stimulated with SARS-CoV-2 spike peptide pools. Freshly drawn whole blood (320 $\mu \mathrm{L}$; drawn within 6 hours of venipuncture) was mixed with $80 \mu \mathrm{L}$ RPMI and stimulated with the indicated SARS-CoV-2 spike peptide pools (Supplemental Table 1 ) at $2 \mu \mathrm{g} / \mathrm{mL}$ or with DMSO as a control. After 16 hours of culturing, the culture supernatant (plasma) was collected and stored at $-80^{\circ} \mathrm{C}$. Cytokine concentrations in the plasma were quantified using an Ella machine with microfluidic multiplex cartridges that measured IFN- $\gamma$ and IL-2 according to the manufacturer's instructions (ProteinSimple). The levels of cytokines present in the plasma of DMSO controls were subtracted from the corresponding peptide pool-stimulated samples. The positivity threshold was set at 10 times the lower limit of quantification of each cytokine (IFN- $\gamma=1.7 \mathrm{pg} / \mathrm{mL}$; IL-2 $=5.4 \mathrm{pg}$ / $\mathrm{mL})$ after DMSO background subtraction.

PBMC isolation. Peripheral blood was collected from all individuals in heparin-containing tubes, and PBMCs from all collected blood sam-

with possible implications for $\mathrm{T}$ cell recognition of the region and $\mathrm{T}$ cell immune escape by emerging viral variants of concern.

In conclusion, we show that the rapid measurement of cytokine production in whole blood after peptide-specific activation is a quick and simple assay that can reliably detect the wide, dynamic range of functionally heterogeneous spike-specific T cell responses induced after vaccination or infection in different individuals. Even though $\mathrm{T}$ cells cannot prevent infection in the absence of antibodies, their pivotal role in the protection from severe disease has been shown in natural infection of healthy individuals $(2,3)$ and oncological patients (7), as well as in vaccinated individuals (16). As such, since the quantity of spike-specific T cells cannot be predicted by the simple measurement of antibodies, this higherthroughput, simple assay can be feasibly performed as part of routine testing to complement existing antibody measurements and thus help to define the correlates of protection necessary for the design of current vaccine strategies.

\section{Methods}

Study participants. Vaccinated individuals were between 21 and 60 years of age, healthy, and had no history of SARS-CoV-2 infection. Wholeblood and serum samples were collected at the indicated intervals for serological and $\mathrm{T}$ cell response analysis. RBD-binding antibodies in the serum of vaccinees V1-V6, before receiving the BNT162b2 vaccine, ples were isolated by Ficoll-Paque density gradient centrifugation.

SARS-CoV-2 spike-specific T cell quantification. The frequency of SARS-CoV-2 spike-specific T cells was quantified as described previously (5). Briefly, freshly isolated or cryopreserved PBMCs (as indicated) were stimulated with SpG peptide pool in an IFN- $\gamma$ ELISPOT assay. ELISPOT plates (MilliporeSigma) were coated with human IFN- $\gamma$ antibody overnight at $4^{\circ} \mathrm{C}$. A total of 400,000 PBMCs were seeded per well and stimulated for 18 hours with the SpG peptide pool at $2 \mu \mathrm{g} / \mathrm{mL}$. The plates were then incubated with a human biotinylated IFN- $\gamma$ detection antibody, followed by streptavidin-alkaline phosphatase (streptavidin-AP) and developed using the KPL BCIP/NBT phosphatase substrate (Seracare Life Sciences). To quantify positive peptide-specific responses, $2 \times$ mean spots of the unstimulated wells were subtracted from the peptide-stimulated wells, and the results were expressed as spot-forming cells (SFC) per $10^{6}$ PBMCs. Results were excluded if negative control wells had more than $30 \mathrm{SFC} / 10^{6}$ PBMCs or if positive control wells (PMA/ionomycin) were negative.

$R B D-h A C E 2$ binding inhibition assay. Antibodies inhibiting the binding of virus to the host cell were measured using a commercial RBD-human angiotensin-converting enzyme 2 (hACE2) binding inhibition assay called cPASS (GenScript). Following the manufacturer's instructions, serum was diluted 1:10 in the kit sample buffer and mixed 1:1 with HRP-conjugated RBD and incubated for 30 minutes at $37^{\circ} \mathrm{C}$. RBD-antibody mixtures were 


\section{All time points analyzed}

Vaccinated ( $n=91 ; 112$ samples)

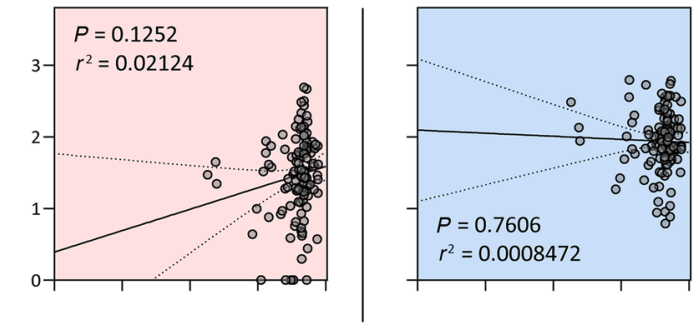

Asymp. COVID-19 ( $n=62 ; 62$ samples)

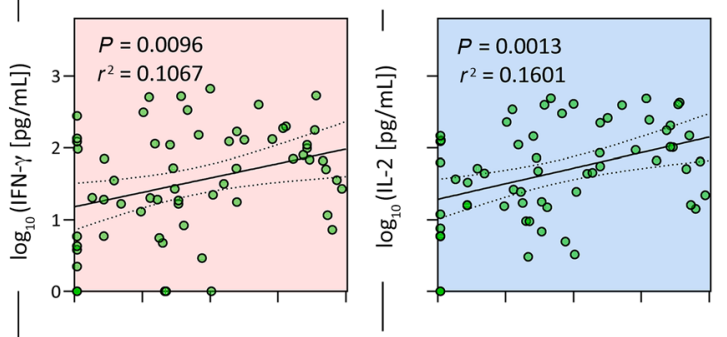

Symp. COVID-19 ( $n=64 ; 107$ samples)

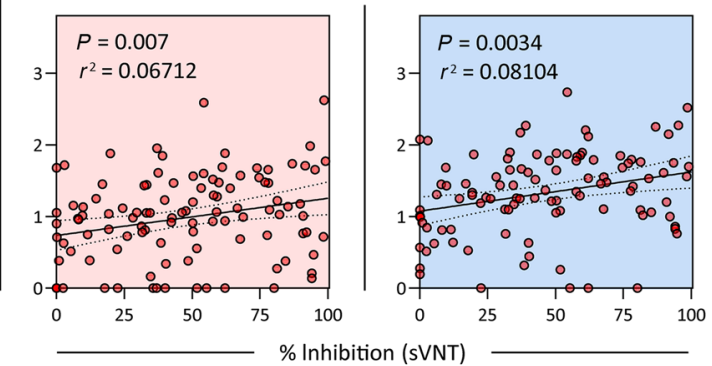

Figure 8. Spike-specific $T$ cell responses do not correlate with neutralizing antibody levels in the serum. Linear regression analysis of IFN- $\gamma$ and IL-2 concentrations in SpG peptide pool-stimulated whole blood and the corresponding SARS-CoV-2 neutralizing capacity of serum from vaccinated individuals ( $n=91 ; 112$ samples) and from asymptomatic ( $n=62$; 62 samples) and symptomatic ( $n=64 ; 107$ samples) COVID-19 patients sampled at all time points available. Dotted lines indicate the $95 \% \mathrm{CI}$. sVNT, surrogate virus neutralization test.

then transferred onto ELISA plates coated with recombinant hACE2 receptor and incubated for 15 minutes at $37^{\circ} \mathrm{C}$. Following incubation, the plates were washed with the kit wash solution followed by incubation with $3,3^{\prime}, 5,5^{\prime}$-tetramethylbenzidine (TMB) substrate for 15 minutes, and the was reaction stopped with stop solution. Absorbance was measured at $\mathrm{OD}_{450} \mathrm{~nm}$. The percentage of inhibition of RBD-hACE2 binding was computed using the following equation: percentage of inhibition $=(1-[(\mathrm{OD}$ of serum + $\mathrm{RBD}) /(\mathrm{OD}$ of negative control $+\mathrm{RBD})]) \times 100$. As recommended in the cPASS kit instructions, a cutoff of $20 \%$ and above was used to determine positive RBD-hACE2 inhibition.

AIM assay. Cryopreserved PBMCs were thawed and stimulated for 24 hours at $37^{\circ} \mathrm{C}$ with the SpG peptide pool $(2 \mu \mathrm{g} / \mathrm{mL})$ in AIM-V media supplemented with $2 \%$ pooled human $A B$ serum. Cells were then stained with the Fixable Yellow Dead Cell Stain Kit (Invitrogen, Thermo Fisher Scientific) followed by staining with the following surface markers as previously described (16): anti-CD3, anti-CD4, antiCD8, anti-CD69, anti-CD134 (OX40), and anti-CD137 (4-1BB). All samples were acquired on a BD-LSR II Analyzer (BD) and analyzed with FlowJo software (BD). The gating strategy is shown in Supplemental Figure 2.

Antibodies. The following antibodies were used in the ELISPOT assay: anti-human IFN- $\gamma$-coating antibody (Mabtech, catalog 3420-31000) and anti-human IFN- $\gamma$ biotin (Mabtech, catalog 3420-6-1000). The following antibodies were used in the AIM assay: anti-human CD3 BV605 (BioLegend, catalog 317321); anti-human CD8 V500 (BD, catalog 560774); anti-human CD4 V650 (BD, catalog 563875); anti-human CD69 AF700 (BioLegend, catalog 310921); anti-human CD134 (OX40) PE (BioLegend, catalog 350004); and anti-human CD137 (4-1BB) APC (BD, catalog 550890).

Statistics. All statistical analyses were performed using GraphPad Prism, version 9 (GraphPad Software). Significant differences in each group were analyzed by 1-way ANOVA with adjustment for multiple comparisons or by the Wilcoxon matched-pairs signed-rank test. Where applicable, the statistical tests used and the definition of center are indicated in the figure legends. Statistical significance was set at a $P$ value of less than 0.05 . In all instances, $n$ refers to the number of patients analyzed.

Study approval. Individuals who had recovered from SARS-CoV-2 infection (asymptomatic: $n=62$; symptomatic: $n=68$ ), were vaccinated with BNT162b2 $(n=112)$, or had SARS-CoV-1 infection 17 years ago $(n=12)$ were enrolled in this study as part of the PROTECT study (National Healthcare Group Domain Specific Review Board [NHG DSRB], ref. 2012/00917); the Healthcare Worker Vaccination study (SingHealth Centralized Institutional Review Board [CIRB], CIRB ref. 2021/2014); the Novel Pathogens study (CIRB ref. 2018/3045); and the SARS Recall study (NHG DSRB ref. 2020/00091). All participants provided written informed consent.

\section{Author contributions}

ATT, NLB, and AB designed the experiments. WNC, RDA, EEO, and LFW performed and analyzed the antibody experiments. JMEL, KK, AC, MDCQ, and NT performed all other experiments and analyzed the data. ATT, NLB, and AB analyzed and interpreted all the data. ATT, NLB, and AB prepared the figures and wrote the manuscript. DY, JXYS, MICC, BEY, LYH, JGHL, and DCL recruited the COVID-19 patients, the SARS-Recall patients, and the vaccinees and provided all clinical samples and data. $\mathrm{AB}$ designed and coordinated the study.

\section{Acknowledgments}

We would like to thank all the clinical and nursing staff who provided care for the patients; all the clinical trial coordinators and staff at the Singapore Infectious Disease Clinical Research Network and the Infectious Disease Research and Training Office, the National Centre for Infectious Diseases, and Singapore General Hospital for their invaluable assistance in coordinating patient recruitment. This study was supported by the Singapore Ministry of Health's National Medical Research Council under its COVID-19 Research Fund (COVID19RF3-0060, COVID19RF-001, and COVID19RF-008).

Address correspondence to: Antonio Bertoletti, Programme in Emerging Infectious Diseases, Duke-NUS Medical School, 8 College Road, Singapore 169857, Singapore. Phone: 65.6601.2646; Email: antonio@duke-nus.edu.sg. 
1. McMahan K, et al. Correlates of protection against SARS-CoV-2 in rhesus macaques. Nature. 2021;590(7847):630-634.

2. Rydyznski Moderbacher C, et al. Antigenspecific adaptive immunity to SARS-CoV-2 in acute COVID-19 and associations with age and disease severity. Cell. 2020;183(4):996-1012.

3. Tan AT, et al. Early induction of functional SARS-CoV-2-specific T cells associates with rapid viral clearance and mild disease in COVID19 patients. Cell Rep. 2021;34(6):108728.

4. Sekine T, et al. Robust T cell immunity in convalescent individuals with asymptomatic or mild COVID-19. Cell. 2020;183(1):158-168.

5 . Le Bert N, et al. Highly functional virusspecific cellular immune response in asymptomatic SARS-CoV-2 infection. JExp Med. 2021;218(5):e20202617

6. Rodda LB, et al. Functional SARS-CoV-2-specific immune memory persists after mild COVID-19. Cell. 2021;184(1):169-183.

7. Bange EM, et al. CD8 ${ }^{+} \mathrm{T}$ cells contribute to survival in patients with COVID-19 and hematologic cancer. Nat Med. 2021;27(7):1280-1289.

8. Walsh EE, et al. Safety and immunogenicity of two RNA-based Covid-19 vaccine candidates. N Engl JMed. 2020;383(25):2439-2450.

9. Jackson LA, et al. An mRNA vaccine against SARS-CoV-2 - preliminary report. $N$ Engl J Med. 2020;383(20):1920-1931.

10. Sahin U, et al. BNT162b2 vaccine induces neutralizing antibodies and poly-specific $\mathrm{T}$ cells in humans [published online May 27, 2021]. Nature. https://doi.org/10.1038/s41586-021-03653-6.

11. Khoury DS, et al. Neutralizing antibody levels are highly predictive of immune protection from symptomatic SARS-CoV-2 infection. Nat Med. 2021;27(7):1205-1211.

12. Chia WN, et al. Dynamics of SARS-CoV-2 neutralising antibody responses and duration of immunity: a longitudinal study. Lancet Microbe. 2021;2(6):e240-e249.

13. Sahin U, et al. COVID-19 vaccine BNT162b1 elicits human antibody and TH1 T cell responses. Nature. 2020;586(7830):594-599.

14. Bonifacius A, et al. COVID-19 immune signatures reveal stable antiviral $\mathrm{T}$ cell function despite declining humoral responses. Immunity.
2021;54(2):340-354.

15. Sherina N, et al. Persistence of SARS-CoV-2specific $\mathrm{B}$ and $\mathrm{T}$ cell responses in convalescent COVID-19 patients 6-8 months after the infection. Med (N Y). 2021;2(3):281-295.

16. Kalimuddin S, et al. Early T cell and binding antibody responses are associated with Covid19 RNA vaccine efficacy onset. $\operatorname{Med}(N Y)$. 2021;2(6):682-688.

17. Wyllie D, et al. SARS-CoV-2 responsive T cell numbers are associated with protection from COVID-19: a prospective cohort study in keyworkers [preprint]. https://doi.org/10.1101/2020.11.02 .20222778. Posted on medRxiv May 02, 2021.

18. Goletti D, et al. Selected RD1 peptides for active tuberculosis diagnosis: comparison of a gamma interferon whole-blood enzyme-linked immunosorbent assay and an enzyme-linked immunospot assay. Clin Diagn Lab Immunol. 2005;12(11):1311-1316.

19. Petrone L, et al. A whole blood test to measure SARS-CoV-2-specific response in COVID-19 patients. Clin Microbiol Infect. 2021;27(2):286. e7-286.e13.

20. Murugesan K, et al. Interferon-gamma release assay for accurate detection of SARS-CoV-2 $\mathrm{T}$ cell response [published online October 9, 2020]. Clin Infect Dis. https://doi.org/10.1093/ cid/ciaa1537.

21. Li J, et al. Safety and immunogenicity of the SARS-CoV-2 BNT162b1 mRNA vaccine in younger and older Chinese adults: a randomized, placebo-controlled, double-blind phase 1 study. Nat Med. 2021;27(6):1062-1070.

22. Ford T, et al. Cryopreservation-related loss of antigen-specific IFN $\gamma$ producing $\mathrm{CD} 4^{+} \mathrm{T}$-cells can skew immunogenicity data in vaccine trials: Lessons from a malaria vaccine trial substudy. Vaccine. 2017;35(15):1898-1906.

23. Tarke A, et al. Comprehensive analysis of T cell immunodominance and immunoprevalence of SARS-CoV-2 epitopes in COVID-19 cases. Cell Rep Med. 2021;2(2):100204

24. Woldemeskel BA, et al. SARS-CoV-2 mRNA vaccines induce broad CD4+ $\mathrm{T}$ cell responses that recognize SARS-CoV-2 variants and $\mathrm{HCoV}-$ NL63. J Clin Invest. 2021;131(10):e149335. 25. Le Bert N, et al. SARS-CoV-2-specific T cell immunity in cases of COVID-19 and SARS, and uninfected controls. Nature. 2020;584(7821):457-462.

26. Reynolds CJ, et al. Prior SARS-CoV-2 infection rescues $\mathrm{B}$ and $\mathrm{T}$ cell responses to variants after first vaccine dose [published online April 30, 2021]. Science. https://doi.org/10.1126/science.abh1282.

27. Snyder TM et al. Magnitude and dynamics of the T-cell response to SARS-CoV-2 infection at both individual and population levels [preprint]. https://doi.org/10.1101/2020.07.31.20165647. Posted on medRxiv August 4, 2020.

28. Mateus J, et al. Low dose mRNA-1273 COVID19 vaccine generates durable T cell memory and antibodies enhanced by pre-existing crossreactive T cell memory [preprint]. https://doi. org/10.1101/2021.06.30.21259787. Posted on medRxiv July 5, 2021.

29. Reynolds CJ, et al. Discordant neutralizing antibody and $\mathrm{T}$ cell responses in asymptomatic and mild SARS-CoV-2 infection. Sci Immunol. 2020;5(54):eabf3698.

30. Dan JM, et al. Immunological memory to SARSCoV-2 assessed for up to 8 months after infection. Science. 2021;371(6529):eabf4063.

31. Chen JS, et al. High-affinity, neutralizing antibodies to SARS-CoV-2 can be made in the absence of $\mathrm{T}$ follicular helper cells [preprint]. https:// doi.org/10.1101/2021.06.10.447982. Posted on bioRxiv June 11, 2021.

32. Doria-Rose N, et al. Antibody persistence through 6 months after the second dose of mRNA-1273 vaccine for Covid-19. N Engl J Med. 2021;384(23):2259-2261.

33. van Doremalen N, et al. ChAdOx1 nCoV-19 vaccine prevents SARS-CoV-2 pneumonia in rhesus macaques. Nature. 2020;586(7830):578-582.

34. Corbett KS, et al. SARS-CoV-2 mRNA vaccine design enabled by prototype pathogen preparedness. Nature. 2020;586(7830):567-571.

35. Bos R, et al. Ad26 vector-based COVID-19 vaccine encoding a prefusion-stabilized SARS-CoV-2 spike immunogen induces potent humoral and cellular immune responses. NPJ Vaccines. 2020;5(1):91-11.

36. Vogel AB, et al. BNT162b vaccines protect rhesus macaques from SARS-CoV-2. Nature. 2021;592(7853):283-289. 